\title{
Über gleichzeitige Abscheidung von Titan und Zirkon bei Gegenwart von Eisen.
}

\author{
Von \\ M. Ditrrich und S. Freund.
}

Vor einiger Zeit hatte der eine ${ }^{1}$ von uns zusammen mit R. PoHI ein Verfahren angegeben, Zirkon neben Titan zu bestimmen, welches darauf beruht, dafs beide Elemente von den gleichzeitig vorhandenen anderen, wie Eisen usw. abgeschieden und zusammen gewogen werden; Titan wird sodann nach Aufschluís des Glührückstandes kolorimetrisch nach W ELLien bestimmt, während $\mathrm{ZrO}_{3}$ sich aus der Differenz berechnen läfst. Die zur Trennung von Titan und Zirkon von Eisen verwendete Methode - Ammoniumsulfid in weinsaurer Lösung - besals infolge der Schwierigkeit der Weinsäurezerstörung manche Mängel, und es war damals schon versucht worden, die Methode durch eine zweckmärsigere zu ersetzen, zumal dies besonders für die Gesteinsanalyse von Wichtigkeit war. Diese Versuche haben wir jetzt fortgesetzt und teilen im Nachstehenden die erhaltenen Resultate mit.

Es war bekannt, dafs Titan bei gleichzeitiger Anwesenheit von Zirkon aus schwach saurer Lösung beim Kochen nur unvollständig, manchmal auch zirkonhaltig, ausfällt, dafs dagegen Titan allein vollständig ausfällbar ist, wenn gowisse Salze, besonders von schwachen Säuren, wie Natriumacetat oder -thiosulfat zugegen sind, durch welche also die starke Säure durch eine schwächere ersetzt wird.

Es wurde deshalb untersucht, ob vielleicht unter derartigen Bedingungen die Ausfällung bei gleichzeitiger Anwesenheit beider Elemente vollständig quantitativ verläuft. In allen Fällen wurden

1 M. Drrtrich und R. Pонц, „Über Bestimmung von Zirkon neben Titan, insbesondere in Gesteinen". $Z$. anorg. Ohem. 43 (1905), 236-241. 
reinste Substanzen zur Analyse verwendet, wie dies bereits früher beschrieben ist ${ }^{1}$.

Bei Gegenwart von Natriumacetat gelingt die Trennung des Titans und Zirkons vom Eisen vollständig, wenn man dafür Sorge trägt, dafs Eisen nur in der Ferroform vorhanden ist. Zur Entfernung des aus dem Platintiegel stammenden Platins und zur gleichzeitigen Reduktion des Eisens leitet man Schwefelwasserstoff in die saure Lösung der Bisulfatschmelze bis zur Sättigung ein und filtriert nach dem Absitzen des Niederschlages in einem $3 / 4$ LiterKolben aus Jenenser Glas; dieser kann mit einem dreifach durchbohrten Stopfen verschlossen werden, durch dessen zwei Öfnungen Gas- $\mathrm{Zu}$ - und Ableitungsröhren gehen und in dessen dritter Durchbohrung ein Tropftrichter eingeführt wird. Das saure Filtrat der Sulfate wird jetzt mit Natriumcarbonat neutralisiert, wobei man die Entstehung einer Dunkelfärbung des sich bildenden Ferrosulfids als Indikator benützen kann. Man setzt dann wieder einige Tropfen verdünnter Schwefelsäure zu, leitet zur völligen Reduktion des Eisens nach Aufsetzens des Stopfens nochmals Schwefelwasserstoff ein und vertreibt diesen schliefslich, indem man zum Sieden erhitzt und gleichzeitig einen mälsig starken Strom ron mit Wasser gewaschener $\mathrm{CO}_{2}$ so lange durchleitet, bis eine verdünnte ammoniakalische Lösung von Nitroprussidnatrium bei Einleiten der austretenden Dämpfe nicht mehr violett gefärbt wird, bis also sämtlicher $\mathrm{H}_{2} \mathrm{~S}$ entfernt jst. $\mathrm{Zu}$ der nun vollkommen ferrisalzfreien und vor Oxydation geschützten heil'sen Flüssigkeit, lälst man durch den Tropftrichter eine konzentrierte wässerige Lösung ron etwa $5 \mathrm{~g}$ Natriumacetat fliefsen und gibt sodann, wenn nötig, durch den Tropftrichter noch soviel heifses Wasser hinzu, dals die Flüssigkeit ca. 400 bis $500 \mathrm{ccm}$ beträgt. Unter weiterem Durchleiten von $\mathrm{CO}_{2}$ und Ersatz des verdampfenden Wassers erhitzt man noch etwa 1 Stunde weiter und lälst schlielslich im $\mathrm{CO}_{2}$-Strom erkalten. Den abgeschiedenen weifsen Niederschlag filtriert man auf ein gröfseres Filter ab und wäscht ihn dort gut mit heilsem, schwach schwefelwasserstoffhaltigem Wasser aus, um Oxydation des Eisens und dadurch erfolgende Mitabscheidung von Eisenoxyd zu vermeiden. Am Kolben noch etwa anhaftendes Titan oder Zirkon bringt man am besten mit einigen Tropfen konzentrierte Schwefelsäure und etwas Wasserstoffsuperoxyd in Lösung; durch Verdünnen mit Wasser und Fällen mit Ammoniak

1 1. c. S. 238 . 
kann auch diese Menge noch erbalten werden. Der Niederschlag wird im Platintiegel verascht und vor dem Gebläse bis zur Gewichtskonstanz geglüht. Nach Schmelzen mit Natriumhydrosulfat $\left(\mathrm{NaHSO}_{4}\right)$ und Auflösen der Schmelze in Wasser wird das in der Lösung etwa vorhandene $\mathrm{SiO}_{2}$ abfiltriert und gewogen, sodann $\mathrm{TiO}_{2}$ colorimetrisch bestimmt und $\mathrm{ZrO}_{2}$ aus der Differenz berechnet. Enthielt die Lösung die richtige Säuremenge und war sie nach dem Einleiten von Schwefelwasserstoff vor Luftzutritt vollkommen geschützt, so ist der mit Natriumacetat erhaltene Niederschlag stets frei von Eisen; ist der Niederschlag gefärbt, so mufs die Fällung wiederholt werden.

Im Filtrat von $\mathrm{TiO}_{2}$ und $\mathrm{ZrO}_{2}$ lälst sich das Natriumacetat, ähnlich wie die Weinsäure, etwa durch Persulfat nur sehr schwer zerstören; man fällt dam besser in der stark mit Salzsäure angesäuerten und eingedampften Flüssigkeit das Eisen nach Oxydation desselben mittels Wasserstoffsuperoxyd und dgl. durch Ammoniak, löst den Niederschlag wieder in Salzsäure und fällt ihn nochmals.

I.

$0.5833 \mathrm{~g} \mathrm{FeSO}_{4}\left(\mathrm{NH}_{4}\right)_{2} \mathrm{SO}_{4} \cdot 6 \mathrm{H}_{2} \mathrm{O}$ (entsprechend $\left.0.1188 \mathrm{~g} \mathrm{Fe}_{2} \mathrm{O}_{3}\right)$ $+0.0895 \mathrm{~g} \mathrm{TiO}_{2}{ }^{1}$ und $0.0524 \mathrm{~g} \mathrm{ZrO}_{2}$ gaben:

$0.1198 \mathrm{~g} \mathrm{Fe}_{2} \mathrm{O}_{3}, 0.0890 \mathrm{~g} \mathrm{TiO}_{2}$ und $0.0521 \mathrm{~g} \mathrm{ZrO}_{2}$.

II.

$0.5938 \mathrm{~g} \mathrm{FeSO}_{4}\left(\mathrm{NH}_{4}\right)_{2} \mathrm{SO}_{4} \cdot 6 \mathrm{H}_{2} \mathrm{O}$ (entsprechend $0.1212 \mathrm{~g} \mathrm{Fe}_{2} \mathrm{O}_{3}$ ) $+0.0711 \mathrm{~g} \mathrm{TiO}_{2}$ und $0.0209 \mathrm{~g} \mathrm{ZrO}_{2}$ gaben:

$0.1221 \mathrm{~g} \mathrm{Fe}_{2} \mathrm{O}_{3}, 0.0717 \mathrm{~g} \mathrm{TiO}_{2}$ und $0.0224 \mathrm{~g} \mathrm{ZrO}_{2}$.

III.

$0.4938 \mathrm{~g} \mathrm{FeSO}_{4}\left(\mathrm{NH}_{4}\right)_{2} \mathrm{SO}_{4} \cdot 6 \mathrm{H}_{2} \mathrm{O}$ (entsprechend $0.1008 \mathrm{~g} \mathrm{Fe}_{2} \mathrm{O}_{3}$ ) $+0.0666 \mathrm{~g} \mathrm{TiO}_{2}$ und $0.0339 \mathrm{~g} \mathrm{ZrO}_{2}$ gaben:

$0.1011 \mathrm{~g} \mathrm{~F}_{2} \mathrm{O}_{3}, 0.0680 \mathrm{~g} \mathrm{TiO}_{2}$ und $0.0339 \mathrm{~g} \mathrm{ZrO}_{2}$.

Bei Gegenwart von Natriumthiosulfat lälst sich sowohl Titan wie auch Zirkon allein in schwach saurer, stark verdünnter Lösung bei Siedebitze von Eisen trennen; Chancel ${ }^{2}$, Stromerer ${ }^{3}$, und

1 Da im Laufe der Arbeit verschieden starke $\mathrm{TiO}_{3}$ und $\mathrm{ZrO}$ - $\mathrm{L}$ ösungen verwendet wurden, sind hier jedesmal nur die angewandten Gewichtsmengen angegeben.

${ }^{2}$ Journ. prakt. Chem. 74, 471.

${ }^{3}$ Ann. Chem. Pharm. 113, 127. 
Hermas ${ }^{1}$ benützten diese Tatsache zur Bestimmung des Zirkoniums neben Eisen. Da auch Titan durch Natriumthiosulfat quantitativ als Titansäure gefällt wird, so war anzunehmen, dafs die Trennung, auch wenn beide Elemente - Titan und Zirkon - gleichzeitig vorhanden waren, möglich sei.

Die nach Reduktion durch Schwefelwasserstoff (s. oben) behandelte Lösung der Sulfatschmelze wird in ein grölseres Becherglas filtriert, mit Natriumcarbonat neutralisiert und unter Einleiten von $\mathrm{CO}_{2}$ so lange erhitzt, bis der $\mathrm{H}_{2} \mathrm{~S}$ wieder vertrieben war.

Nach Verdünnen auf etwa $400 \mathrm{ccm}$ wird unter weiterem $\mathrm{CO}_{2}$ Einleiten und Ersatz des verdampfenden Wassers eine Lösung von etwa $5 \mathrm{~g}$ Natriumthiosulfat hinzugegeben und das Ganze noch $1 \mathrm{bis}$ 2 Stunden erwärmt, bis keine $\mathrm{SO}_{2}$ mehr weggeht. Der ausfallende flockige, weifse Niederschlag, welchem etwas Schwefel beigemengt ist, erwies sich bei der Prüfung als vollkommen frei von Eisen; er läfst sich leicht nach dem Absitzen und Erkalten oder besser noch nach längerem Stehen filtrieren, ohne dafs Schwefel durchs Filter geht. Das Veraschen des gut ausgewaschenen Niederschlages kann im Platintiegel vorgenommen werden, der bei langsamem und vorsichtigem Erhitzen von dem verbrennenden Schwefel nicht beschädigt wird. Nach dem Erhitzen mit Ammoniumcarbonat, um Spuren von Sulfaten, die hartnäckig zurückgehalten werden, zu vertreiben, und Glühen des Niederschlages bis zur Gewichtskonstanz vor dem Gebläse wird die Summe der reinen Oxyde von Titan und Zirkonium erhalten und nach Schmelzen mit $\mathrm{NaHSO}_{4}$, Auflösen in Wasser, durch Subtraktion der mit Wasserstoffsuperoxyd colorimetrisch bestimmten Titansäure das Gewicht der Zirkonerde gefunden, wie ober bei der Natriumacetatmethode angegeben.

Im Filtrate wurde das Eisentetrathionat und das unverändert gebliebene Thiosulfat durch Kochen mit starker Salpetersäure zerstört und das Erhitzen so lange fortgesetzt, bis der anfangs milchig ausgeschiedene Schwefel sich zusammengeballt hatte und dann gut filtriert werden konnte. Das Eisen kann nun, wie gewöhnlich, durch Ammoniak abgeschieden und als $\mathrm{Fe}_{2} \mathrm{O}_{3}$ gewogen werden.

I.

$0.5341 \mathrm{~g} \mathrm{FeSO}_{4}\left(\mathrm{NH}_{4}\right) \mathrm{SO}_{4} \cdot 6 \mathrm{H}_{2} \mathrm{O}$ (entsprechend $0.1091 \mathrm{~g} \mathrm{Fe}_{2} \mathrm{O}_{3}$ ) $+0.1075 \mathrm{~g} \mathrm{TiO}_{2}$ und $0.0209 \mathrm{~g} \mathrm{ZrO}_{2}$ gaben:

$0.1097 \mathrm{~g} \mathrm{Fe}_{2} \mathrm{O}_{3}, 0.1065 \mathrm{~g} \mathrm{TiO}_{2}$ und $0.0226 \mathrm{~g} \mathrm{ZrO}_{2}$.

${ }^{3}$ Journ. prakt. Chem. 97, 330. 
II.

$0.5110 \mathrm{~g} \mathrm{FeSO}_{4}\left(\mathrm{NH}_{4}\right)_{2} \mathrm{SO}_{4} .6 \mathrm{H}_{2} \mathrm{O}$ (entsprechend $0.1043 \mathrm{~g} \mathrm{Fe}_{2} \mathrm{O}_{3}$ ) $+0.0896 \mathrm{~g} \mathrm{TiO}_{2}$ und $0.0209 \mathrm{~g} \mathrm{ZrO}_{2}$ gaben:

$$
0.1042 \mathrm{~g} \mathrm{Fe}_{2} \mathrm{O}_{3}, 0.0890 \mathrm{~g} \mathrm{TiO}_{2} \text { und } 0.0926 \mathrm{~g} \mathrm{ZrO}_{2} \text {. }
$$

\section{III.}

$0.5203 \mathrm{~g} \mathrm{FeSO}_{4}\left(\mathrm{NH}_{4 / 2} \mathrm{SO}_{2} \cdot 6 \mathrm{H}_{2} \mathrm{O}\right.$ entsprechend $\left.0.1063 \mathrm{~g} \mathrm{Fe}_{2} \mathrm{O}_{3}\right)$ $+0.0717 \mathrm{~g} \mathrm{TiO}_{2}$ und $0.0419 \mathrm{~g} \mathrm{ZrO}_{2}$ gaben:

$$
0.1066 \mathrm{~g} \mathrm{Fe}_{2} \mathrm{O}_{3}, 0.0715 \mathrm{~g} \mathrm{TiO}_{2} \text { und } 0.0408 \mathrm{~g} \mathrm{ZrO}_{2} \text {. }
$$

Auch in einer Lösung von schwefligor Säure lassen sich Titan und Zirkon gemeinsam abscheiden und von Eisen und Mangan trennen.

Zur Trennung des Titans vom Eisen mittels $\mathrm{SO}_{2}$ haben schon E. HrNTZ und H. WEBER ${ }^{1}$, BASKERviLLE ${ }^{2}$ und $J_{\text {AS. Brakes }}{ }^{3}$ verschiedene Modifikationen empfohlen. Auch Zirkonsalze werden in schwefligsaurer Lösung quantitativ gefällt, da jedoch der dadurch in schwefligsaurer Lösung gebildete Niederschlag kaum filtrierbar ist, erwies es sich als vorteilhafter, die Fällung in salzsaurer Lösung vorzunehmen; der so erhaltene Niederschlag ist flockig und läfst sich sehr leicht filtrieren.

Zur Überführung in Chloride werden am besten die Oxyde mit Natriumcarbonat geschmolzen und die Schmelze in verdünnter Salzsäure gelöst. Die erhaltene stark salzsaure Lösung wird zur Reduktion des Eisens mit schwefliger Säure versetzt, einige Zeit erwärmt und die farblos gewordene Lösung sodann so lange erhitzt, bis kein Geruch nach $\mathrm{SO}_{2}$ mehr wahrzunehmen ist. Nach dem Erkalten neutralisiert man die Flüssigkeit mit Ammoniak und löst einen eventuell ausfallenden Niederschlag durch wenige Tropfen verdünnte Salzsäure wieder auf. Diese nahezu neutrale Lösung wird nun mit ca. $50 \mathrm{ccm}$ einer gesättigten Lösung von schwefliger Säure versetzt und unter Durchleiten eines Kohlensäurestromes und Ersatz des verdampfenden Wassers in dem oben beschriebenen Kolben etwa 1-2 Stunden erhitzt, bis kein Geruch nach schwefliger Säure mehr wahrzunehmen ist und der ausgefallene Niederschlag sich nicht mehr

1 Zeitschr. analyt. Chem. 31, 562.

* Journ. Am. Ohem. Soc. 16, 427.

s Chem. Soc. Ind. S. 18, 1097. 
vermehrt. Derselbe wird heifs abfiltriert; man wäscht ihn gut aus, verascht ihn nafs im Platintiegel und gliuht ihn bis zur Gewichtskonstanz; der Glührückstand war rein wejls und enthielt höchstens nur Spuren von Eisen. Die Bestimmung von $\mathrm{TiO}_{2}$ und $\mathrm{ZrO}_{2}$ erfolgte darin wie früher. Zur Kontrolle der vollständigen Ausfällung neutralisiert man das klare Filtrat nochmals mit Ammoniak und prüft durch längeres Kochen mit einer Lösung von schwefliger Säure, ob noch Niederschlag entsteht.

Im Filtrate konnte das Eisen nach Oxydation mit Wasserstoffsuperoxyd oder Bromwasser und dgl. durch Ammoniak in der Hitze gefällt und in $\mathrm{Fe}_{2} \mathrm{O}_{3}$ übergefïhrt werden.

I.

$0.5540 \mathrm{~g} \mathrm{FeSO}_{4}\left(\mathrm{NH}_{4}\right)_{2} \mathrm{SO}_{4} \cdot 6 \mathrm{H}_{2} \mathrm{O}$ (entsprechend $0.1131 \mathrm{~g} \mathrm{Fe}_{2} \mathrm{O}_{3}$ ) $+0.0842 \mathrm{~g} \mathrm{TiO}_{2}$ und $0.0471 \mathrm{~g} \mathrm{ZrO}_{2}$ gaben:

$0.1136 \mathrm{~g} \mathrm{Fe}_{2} \mathrm{O}_{3}, 0.0836 \mathrm{~g} \mathrm{TiO}_{2}$ und $0.0465 \mathrm{~g} \mathrm{ZrO}_{2}$.

II.

$0.5021 \mathrm{~g} \mathrm{FeSO}_{4}\left(\mathrm{NH}_{4}\right)_{2} \mathrm{SO}_{4} \cdot 6 \mathrm{H}_{2} \mathrm{O}$ (entsprechend $0.1025 \mathrm{~g} \mathrm{Fe}_{2} \mathrm{O}_{3}$ ) $+0.0754 \mathrm{~g} \mathrm{TiO}_{2}$ und $0.0358 \mathrm{~g} \mathrm{ZrO}_{2}$ gaben:

$0.1027 \mathrm{~g} \mathrm{Fe}_{2} \mathrm{O}_{3}, 0.0750 \mathrm{~g} \mathrm{TiO}_{2}$ und $0.0353 \mathrm{~g} \mathrm{ZrO}_{2}$.

III,

$0.5532 \mathrm{~g} \mathrm{FeSO}_{4}\left(\mathrm{NH}_{4}\right)_{2} \mathrm{SO}_{4} \cdot 6 \mathrm{H}_{2} \mathrm{O}$ (entsprechend $0.1129 \mathrm{~g} \mathrm{Fe}_{2} \mathrm{O}_{3}$ ) $+0.0643 \mathrm{~g} \mathrm{TiO}_{2}$ und $0.0284 \mathrm{~g} \mathrm{ZrO}_{2}$ gaben:

$0.1136 \mathrm{~g} \mathrm{Fe}_{2} \mathrm{O}_{3}, 0.0641 \mathrm{~g} \mathrm{TiO}_{2}$ und $0.0276 \mathrm{~g} \mathrm{ZrO}_{2}$.

Verhältnismälsig am einfachsten erfolgt die Abscheidung von Titan und Zirkonium und die gleichzeitige Trennung von Eisen und Mangan, wenn man die durch Schwefelwasserstoff reduzierte und wie oben beinahe neutralisierte Lösung unter Zugabe von etwa $\overline{\mathbf{0}}-10 \mathrm{~g}$ Ammoniumsulfat oder einer entsprechenden Menge einer konzentrierten Lösung davon im Kohlensäurestrom längere Zeit kocht; infolge des hydrolisierenden Einflusses werden die in der schwach sauren Flüssigkeit vermutlich kolloidal in Lösung befindlichen Titanund Zirkonverbindungen zerlegt und fallen als Oxyde aus. Die Ausführung der Trennung erfolgt genau so wie früher angegeben. Der entstandene Niederschlag ist leicht filtrierbar; er wird mit heifsem Wasser gut ausgewaschen, im Platintiegel verascht und bis zur Gewichtskonstanz vor dem Gebläse geglüht. Die Bestimmung des 
Titans und Zirkoniums wird in der gleichen Weise, wie bei den vorigen Trennungen vorgenommen. - Im Filtrate kann das Eisen nach der Oxydation mit Wasserstoffsuperoxyd durch Ammoriak in der Hitze wie gewöhnlich bestimmt werden.

Dies Verfahren ergibt, wie die folgenden Analysen zeigen, gute Resultate. Vor dem Verfahren mit Natriumacetat hat es den Vorteil, dal's hier die bei der Fällung des Eisens störende Essigsäure in Wegfall kommt, und dals die hydrolytische Wirkung des Ammoniumsulfats auf Eisensalze viel geringer ist, als die des Natriumacetats.

I.

$0.5230 \mathrm{~g} \mathrm{FeSO}_{4}\left(\mathrm{NH}_{4} / 2 \mathrm{SO}_{4} \cdot 6 \mathrm{H}_{2} \mathrm{O}\right.$ (entsprechend $0.1067 \mathrm{~g} \mathrm{Fe}_{2} \mathrm{O}_{3}$ ) $+0.0480 \mathrm{~g} \mathrm{TiO}_{2}$ und $0.0648 \mathrm{~g} \mathrm{ZrO}_{2}$ gaben:

$0.1075 \mathrm{~g} \mathrm{Fe}_{3} \mathrm{O}_{3}, 0.0472 \mathrm{~g} \mathrm{TiO}_{2}$ und $0.0658 \mathrm{~g} \mathrm{ZrO}_{2}$.

II.

$0.5540 \mathrm{~g} \mathrm{FeSO}_{4}\left(\mathrm{NH}_{4}\right)_{2} \mathrm{SO}_{4} \cdot 6 \mathrm{H}_{2} \mathrm{O}$ (entsprechend $0.1131 \mathrm{~g} \mathrm{Fe}_{2} \mathrm{O}_{3}$ ) $+0.0240 \mathrm{~g} \mathrm{TiO}_{2}$ und $0.0412 \mathrm{~g} \mathrm{ZrO}_{2}$ gaben:

$0.1124 \mathrm{~g} \mathrm{Fe}_{2} \mathrm{O}_{3}, 0.0235 \mathrm{~g} \mathrm{TiO}_{2}$ und $0.0406 \mathrm{~g} \mathrm{ZrO}_{2}$.

III.

$0.5204 \mathrm{~g} \mathrm{FeSO}_{4}\left(\mathrm{NH}_{4}\right)_{2} \mathrm{SO}_{4} \cdot 6 \mathrm{H}_{2} \mathrm{O}$ (entsprechend $0.1062 \mathrm{~g} \mathrm{Fe}_{2} \mathrm{O}_{3}$ )

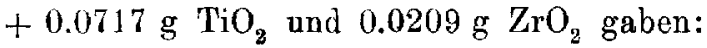

$$
0.1065 \mathrm{~g} \mathrm{Fe}_{2} \mathrm{O}_{3}, 0.0709 \mathrm{~g} \mathrm{TiO}_{2} \text { und } 0.0217 \mathrm{~g} \mathrm{ZrO}_{2} \text {. }
$$

Auch bei alleiniger Gegenwart des Titans neben Eisen ist es empfehlenswert, Ammoniumsulfat zur Trennung zu verwenden, da hierdurch die Fällung in viel kürzerer Zeit als durch blolses Auskochen, wie es bisher üblich war, erfolgt und das lästige Festhalten der gefällten Titansäure an den Glaswänden nicht eintritt.

Auf diese Weise gestaltet sich die namentlich für Gesteinsanalysen wichtige Abscheidung des Titans und auch des Zirkons von Eisen und Mangan, nachdem durch die vorausgegangene Natronschmelze Aluminium und Phosphorsäure entfernt sind, äufserst einfach und leicht ausführbar.

Feidelberg, Privatlaboratorium von Prof. M. Dittrich.

Bei der Redaktion eingegangen am 7. November 1907. 